Maria Salete Medeiros Vieira

Elisa Camargo Siebert ${ }^{2}$

Wi uam Queroz Guimarães WieGandt CeGlo²

Meire Hellen de Almeira ${ }^{2}$

Tatyana Stenger Batista ${ }^{3}$

Paulo Fontoura Freitas ${ }^{4}$

Artigo Original

Palavras-chave

Natimorto

Morte fetal/prevenção \& controle

Causas de morte

Autopsia

Keywords

Stillbirth

Fetal death/prevention \& control Cause of death

Autopsy

\title{
Dificuldades para a identificação da causa do óbito fetal: como resolver?
}

\section{Difficulties for identification of cause of fetal death: how to solve?}

\section{Resumo}

OBJETIVO: Identificar as causas dos óbitos fetais (OF) e avaliar as causas deste desfecho. Propor o emprego do sistema Relevant Condition of Death (ReCoDe) na elucidação das causas de OF para minimizar o número de causas desconhecidas. MÉTODOS: Estudo transversal relacionado às mortes fetais atendidas em um hospital universitário especializado do Sul do Brasil, de Janeiro de 2000 a dezembro de 2009. Os dados foram coletados das Declarações de Óbito, dos prontuários maternos e dos laudos de estudo dos fetos e anexos, sendo os achados comparados. A análise dos dados foi realizada pelo programa SPSS versão 17.0. RESULTADOS: Foram incluídos neste estudo 111 fetos e suas respectivas mães. A comparação entre o diagnóstico das causas encontradas nos laudos do exame dos fetos e anexos e o diagnóstico clínico foi de $74(66,7 \%)$ e $73(65,8 \%)$, respectivamente. Em 48,7\% dos casos, ambas encontraram uma potencial causa e 16,2\% permaneceram desconhecidas. Utilizando-se a classificação ReCoDe, somente 9,9\% dos OFs ficaram como "não classificados". CONCLUSÕES: Os laudos da autópsia fetal e exame anatomopatológico e as avaliações clínicas não apresentaram diferenças significativas na identificação da causa do OF. Ao se comparar os resultados do ReCoDe com as causas de óbito da clínica/patologia, verificou-se que ela é uma ferramenta importante no esclarecimento da causa mortis, reduzindo a quantidade daqueles OFs que permaneceram sem uma possível etiologia.

\section{Abstract}

PURPOSE: To identify the causes of fetal death in the studied population and to measure their contribution in identifying the cause of this outcome. To propose the use of the system Relevant Condition of Death (ReCoDe) in elucidating the causes of fetal death to minimize the number of unknown causes. METHODS: Cross-sectional study related to fetal deaths seen at a specialized academic hospital in the South of Brazil, from January 2000 to December 2009. The data were collected in the death certificates, maternal medical records and the reports of study of fetuses and attachments, and the findings were compared. Data analysis was performed using SPSS version 17.0. RESULTS: Were included 111 fetuses and their respective mothers in this study. The comparison between the diagnostic causes in the pathology and clinical evaluation showed $74(66.7 \%)$ and $73(65.8 \%)$, respectively. Together, they found a potential cause in $48.7 \%$ of cases, while $16.2 \%$ remained unknown. When analyzing both together with the ReCoDe system, only $9.9 \%$ of stillbirths remained as "unclassified." CONCLUSIONS: The proportion of diagnoses in the cause of death among the pathological and clinical evaluation showed no significant difference. When comparing the results of the cause of death suggested by the clinic/pathology with the use of the ReCoDe system, it appears that this tool has helped to clarify the cause by reducing the amount of those that remained without a possible etiology.

Correspondêncio

Maria Salete Medeiros Vieir Hospital Universitário Polydoro Ennani de São Thiago - Divisão de Tocoginecologia - Universidade Federal de Santa Catarin Campus Universitário, $\mathrm{S} / \mathrm{n}^{0}$ - Trindade - Caixa Postal: 5199 Florianópolis (SC), Brasil

Recebido

27/02/2012

Aceito com modificacōôs

$21 / 08 / 2012$
Trabalho realizado no Hospital Universitário Polydoro Ernani de São Thiago, Universidade Federal de Santa Catarina - UFSC Florianópolis (SC), Brasil.

'Departamento de Ginecologia e Obstetrícia e Hospital Universitário da Universidade Federal de Santa Catarina - UFSC - Florianópolis (SC), Brasil. ${ }^{2}$ Curso Acadêmico de Medicina da Universidade Federal de Santa Catarina - UFSC - Florianópolis (SC), Brasil.

${ }^{3}$ Serviço de Ginecologia e Obstetrícia do Hospital Universitário da Universidade Federal de Santa Catarina - UFSC - Florianópolis (SC), Brasil. 4Programa de Pós-Graduação em Ciências Médicas e Serviço de Saúde Pública do Hospital Universitário da Universidade Federal de Santa Catarina - UFSC - Florianópolis (SC), Brasil.

Conflito de interesses: não há 


\section{Introdução}

O óbito fetal (OF) é uma das mais frequentes ocorrências adversas durante a gestação $0^{1,2}$. Ilustrando a magnitude do assunto, a Organização Mundial da Saúde (OMS) estimou, somente para o ano de 2009, o total de óbitos fetais em 2,6 milhões, com taxa de mortalidade fetal de 18,9 óbitos fetais para cada 1 mil nascidos vivos ${ }^{3}$. Para o mesmo ano, o Brasil registrou 39.429 óbitos fetais e 11,15 para cada $1 \mathrm{mil}^{4}$. Quando comparados às principais causas de morte globais em todas as faixas etárias, os OFs estariam em quinto lugar, antes de diarreia, HIV/AIDS, tuberculose, acidentes de trânsito e qualquer tipo de câncer ${ }^{5}$.

O OF é considerado potencialmente evitável, de maneira que ações bem conduzidas são fundamentais e podem diminuir efetivamente as taxas de mortalidade. Entretanto, diversas são as dificuldades a serem sanadas ${ }^{6-8}$; a começar pela pouca visibilidade nas políticas globais, visto que os natimortos sequer foram incluídos nos Objetivos de Desenvolvimento do Novo Milênio pela Organização das Nações Unidas ${ }^{8}$. Em nosso país, não há, nos serviços de saúde, rotinas de análise da ocorrência de OF nem investimentos específicos adequados para a sua redução ${ }^{7,9}$.

Outro problema bastante importante, e uma realidade presente em todo o país, é a baixa qualidade das informações nas declarações de óbito devido a campos não preenchidos e, principalmente, às imprecisões na declaração da "causa da morte"

Mesmo quando investigado o óbito, há, ainda, a dificuldade classificatória, visto que existem mais de 30 sistemas com esta finalidade ${ }^{8,10}$ e que o OF não está associado a uma única etiologia, mas sim é o resultado final de múltiplos fatores de risco e potenciais etiologias ${ }^{11}$. Tal realidade resulta na dificuldade de conhecer e determinar todos os fatores que influenciam e/ou causam a morte dos fetos, tornando difícil a elaboração de ações de intervenção ${ }^{6}$.

Esquemas recentes foram elaborados para permitir a classificação mais eficiente, diminuindo a quantidade de casos de causa desconhecida. Entre eles encontra-se o Relevant Condition at Death $(\mathrm{ReCoDe})^{10}$, que visa identificar condições adversas presentes na gestação, e não necessariamente a sua etiologia. Por conseguinte, permite a utilização de múltiplas categorias de maneira hierárquica. Tem ganhado atenção em detrimento da tradicional classificação de Wigglesworth, ainda recomendada pela OMS e pelo Ministério da Saúde (MS), por permitir classificar um número maior de fetos em condições relevantes ${ }^{11}$. A identificação das causas possibilitaria a sistematização de condutas, ação que poderia diminuir a prevalência desse desfecho e reduzir o risco do mesmo em uma gestação subsequente ${ }^{10-12}$.

Apesar da importância destacada, poucos são os estudos brasileiros disponíveis sobre o óbito intraútero, reflexo da baixa visibilidade, interesse e compreensão de que esse evento é, em grande parte, prevenível ${ }^{9}$. Diante do exposto, objetivou-se, com esta pesquisa, identificar as causas dos OFs na população atendida pelo Hospital Universitário Polydoro Ernani de São Thiago, da Universidade Federal de Santa Catarina (HU/UFSC), e mensurar a contribuição de cada fonte de dados pesquisada para a identificação do que leva ao desfecho, e finalmente propor o emprego do sistema ReCoDe na elucidação dos motivos de óbito para minimizar o número de causas desconhecidas.

\section{Métodos}

Realizou-se um estudo transversal relacionado às mortes fetais atendidas na Maternidade do HU/UFSC, em Florianópolis (SC), no período de janeiro de 2000 a dezembro de 2009.

Foram estabelecidos como critérios de inclusão: fetos natimortos cujas mães foram atendidas no HU/UFSC no período do estudo, com idade gestacional igual ou superior a 22 semanas de gravidez ou peso superior a $500 \mathrm{~g} \mathrm{e}$ que foram encaminhados juntamente com suas placentas para estudo no Serviço de Anatomia Patológica (SAP).

No período, foram assistidas 171 gestantes cuja gestação cursou com OF, totalizando 173 óbitos. Enviaram-se à necropsia apenas 121 fetos, visto que na instituição não há protocolo de investigação que oriente que todos os OFs devam ser estudados e também que alguns pais não consentem a realização desse método investigativo. Do total, 111 fetos foram incluídos na pesquisa, 5 excluídos pelo não envio da placenta para o estudo anatomopatológico e outros 5 , pela idade gestacional ser inferior a 22 semanas ou indeterminada.

Foram estudados os dados contidos nas Declarações de Óbito (DOs), nos prontuários maternos, armazenados no Serviço de Arquivo Médico (SAME), e nos laudos das necropsias dos fetos e anatomopatológico dos anexos, extraídos dos arquivos do Serviço de Anatomia Patológica (SAP) — os achados foram comparados.

A coleta de informações foi realizada por meio de uma ficha previamente elaborada pelos pesquisadores contendo as variáveis clínico-epidemiológicas maternas: idade, etnia, estado civil e escolaridade (em anos de estudo). Ainda foram pesquisados o histórico reprodutivo e a gestação atual (número de gestações, número de OFs prévios e idade gestacional). O OF foi classificado de acordo com a idade gestacional em precoce ou tardio.

O estudo do feto (autópsia) e o anatomopatológico dos anexos foram considerados padrão de referência para a identificação da causa do óbito ${ }^{1,11,13,14}$.

As informações relevantes ao OF foram organizadas em três categorias com suas respectivas variáveis: declaração de óbito; achados clínicos; e estudo dos fetos e anexos. Os subgrupos ordenados dentro de cada categoria foram nominados de acordo com a $10^{\circ}$ Revisão 
da Classificação Estatística Internacional de Doenças e Problemas Relacionados à Saúde (CID-10) ${ }^{15}$ :

- Variáveis clínicas: malformação (Q00-Q99), infecção materna (P00.2), descolamento prematuro de placenta (DPP — P02.1), doença hipertensiva específica da gestação (DHEG - P00.0), alterações do cordão $(\mathrm{P} 02,5)$, diabetes mellitus gestacional (DMG O24.4), outros achados e sem achados;

- Variáveis do estudo fetal e anexos: malformação (Q00-Q99), achados sugestivos de infecção (corioamnionite - P00.2, com infecção fetal associada ou não - P35-P39), hematoma retroplacentário (compatível com DPP — P02.1), sinais de hipóxia (P20.9), alterações placentárias (disfunção, infarto ou insuficiência — P02.2), alterações de cordão (P02.5), outros achados e sem achados;

- Variáveis DO: malformação (Q00-Q99), DPP (P02.1), alterações de cordão (P02.5), hipóxia (P20.9), outras, na dependência de exames complementares, declaração em branco e declaração ausente no prontuário.

Posteriormente, as causas dos OFs foram classificadas de acordo com o sistema ReCoDe, que, após a análise de múltiplas classificações, foi o escolhido para o uso nesta pesquisa. Ele é composto por nove categorias hierárquicas relacionadas a condições fetais, de cordão umbilical, placentárias, amnióticas, uterinas, maternas, intraparto, traumática e não classificado. Quando mais de uma categoria foi encontrada, o feto foi classificado pela mais relevante (classificação primária), seguido pela segunda condição mais relevante (classificação secundária) ${ }^{11}$.

Os dados foram digitados no programa EpiData e analisados no SPSS versão 17.0. Foram comparadas as proporções de diagnósticos clínicos com os laudos dos exames do feto e anexos, aplicando-se o teste $\chi^{2}$ no nível de confiança de $95 \%$, sendo apresentado o intervalo de confiança e o valor $\mathrm{p}$.

Este estudo foi submetido e aprovado pelo Comitê de Ética em Pesquisa com Seres Humanos da UFSC, sob $\mathrm{n}^{\mathrm{o}} 816$ e seguindo os princípios éticos da pesquisa envolvendo seres humanos, resolução 196/96 do Conselho Nacional de Saúde.

\section{Resultados}

$\mathrm{Na}$ amostra selecionada, a idade das gestantes variou entre 15 e 42 anos, com média de $23,9( \pm 6,4)$ anos. A maioria delas $(88,6 \%)$ era caucasiana e $73,6 \%$ eram casadas ou viviam em união estável. Ensino fundamental incompleto foi identificado em $45,1 \%$ das mulheres. A média de gestações prévias foi de $2,5( \pm 1,9)$, variando entre nenhuma a 13 gestações, com mediana de uma gestação. Foi identificado OF prévio em 6,3\%. Dos 111 fetos, $63 \%$ tiveram óbito tardio (Tabela 1 ).
Em relação à DO, 47,7\% (n=53) encontravam-se na dependência de exames complementares e 1,8\% ( $\mathrm{n}=2)$ estava em branco. Quando foi apontada causa de óbito, as mais prevalentes foram hipóxia $(18,9 \%$ do total dos óbitos, $\mathrm{n}=21)$ e malformação $(7,2 \%, \mathrm{n}=8)$.

As principais causasde morte determinados pelo exame do feto e anexos foram os achados sugestivos de infecção (corioamnionite, com infecção fetal associada ou não), correspondendo a $17,1 \%$ dos casos, seguidos por malformações fetais e alterações placentárias, ambas com frequência de $14,4 \%$, cada. Os fatores somam $66,6 \%$ de casos que tiveram uma provável causa de óbito, permanecendo 33\% sem causa definida (Tabela 2).

Tabela 1. Características clínicas e sociodemográficas em gestantes cujas gestações evoluíram para óbito fetal, atendidas no Hospital Universitário da Universidade Federal de Santa Catarina, em Florianópolis, entre janeiro de 2000 e dezembro de 2009 ( $n=111$ )

\begin{tabular}{|c|c|c|}
\hline Variáveis & Frequência & $\%$ \\
\hline \multicolumn{3}{|l|}{ Idade (anos) } \\
\hline$\leq 19$ & 16 & 14,4 \\
\hline $20-34$ & 81 & 73,0 \\
\hline$\geq 35$ & 14 & 12,6 \\
\hline \multicolumn{3}{|l|}{ Raça* } \\
\hline Caucasiana & 93 & 88,6 \\
\hline Não-caucasiana & 12 & 11,4 \\
\hline \multicolumn{3}{|l|}{ Estado civil** } \\
\hline Solteira & 23 & 21,0 \\
\hline Casada/União estável & 81 & 73,6 \\
\hline Separada/Divorciada & 4 & 3,6 \\
\hline Viúva & 2 & 1,8 \\
\hline \multicolumn{3}{|l|}{ Escolaridade ${ }^{* * *}$} \\
\hline Analfabeta & 2 & 2,5 \\
\hline $1-3$ anos & 9 & 11,0 \\
\hline 4-7 anos & 28 & 34,1 \\
\hline $8-11$ anos & 32 & 39,0 \\
\hline$\geq 12$ anos & 11 & 13,4 \\
\hline \multicolumn{3}{|l|}{ Número de gestacões } \\
\hline Primigesta & 43 & 38,7 \\
\hline Secundigesta & 22 & 19,8 \\
\hline Multigesta & 46 & 41,5 \\
\hline \multicolumn{3}{|l|}{ Óbito fetal } \\
\hline Precoce & 41 & 37,0 \\
\hline Tardio & 70 & 63,0 \\
\hline \multicolumn{3}{|l|}{ Óbito fetal prévio } \\
\hline Não & 104 & 93,7 \\
\hline Sim & 7 & 6,3 \\
\hline
\end{tabular}

Tabela 2. Informações relevantes ao óbito fetal encontradas pelo estudo dos fetos e anexos submetidos a autópsia no Hospital Universitário da Universidade Federal de Santa Catarina, em Florianópolis, de janeiro de 2000 a dezembro de 2009

\begin{tabular}{|c|c|c|}
\hline Variáveis & Frequência & $\%$ \\
\hline Achados sugestivos de infecção & 19 & 17,1 \\
\hline Malformações fetais & 16 & 14,4 \\
\hline Disfunção, infarto ou insuficiência placentária & 16 & 14,4 \\
\hline Hematoma retroplacentário & 8 & 7,2 \\
\hline Sinais de hipóxia & 5 & 4,5 \\
\hline Alterações no cordão umbilical & 3 & 2,7 \\
\hline Outros achados & 7 & 6,3 \\
\hline Sem achados & 37 & 33,4 \\
\hline Total & 111 & 100,0 \\
\hline
\end{tabular}


Quanto à avaliação clínica, 73 casos $(65,8 \%)$ apresentam uma potencial causa, sendo as clínicas as mais incidentes: malformações $(26,0 \%, \mathrm{n}=19)$, DHEG $(16,4 \%, \mathrm{n}=12)$, $\operatorname{DPP}(15,1 \%, \mathrm{n}=11)$ e infecção materna $(11,0 \%, \mathrm{n}=8)$. Em $34,3 \%$ não foi encontrada causa pela avaliação (Tabela 3).

Ao se comparar os diagnósticos do estudo dos fetos e anexos com a avaliação clínica, os achados não diferiram estatisticamente, sendo $74(66,7 \%)$ e $73(65,8 \%)$, respectivamente, com correlação positiva em $48,6 \%$ dos casos, permanecendo desconhecidos 16,2\% deles (Tabela 4).

As causas dos óbitos fetais foram também analisadas de acordo com a classificação ReCoDe. Neste sistema, na classificação primária as alterações fetais foram as mais prevalentes, totalizando $58,6 \%$ dos casos. Destes, a principal categoria foi a restrição de crescimento intrauterino (RCIU), responsável por $55,4 \%$ das alterações fetais e $32,5 \%$ de todos os OF $(n=36)$, seguido por malformação congênita, com 24,6 e $17,1 \%$ do total $(n=19)$, respectivamente. O grupo dos achados placentários perfizeram $25,2 \%$ do total, sendo as categorias mais prevalentes o descolamento prematuro de placenta $(23,1 \%)$ e a insuficiência placentária $(18,5 \%)$. Em $7,2 \%$ dos casos não foi identificada relação relevante com o desfecho, e em $2,7 \%$ dos óbitos fetais não havia informação acerca do mesmo no prontuário, portanto, $9,9 \%$ dos casos permaneceram como não classificados.

Identificou-se uma classificação secundária em 64,0\% $(\mathrm{n}=71)$. As categorias secundárias mais prevalentes foram

Tabela 3. Informações relevantes ao óbito fetal, podendo justificá-lo, encontradas pela avaliação clínica dos fetos e anexos cujas mães foram atendidas no Hospital Universitário da Universidade Federal de Santa Catarina, em Florianópolis, de janeiro de 2000 a dezembro de 2009 ( $n=111)$

\begin{tabular}{lcc}
\hline Variáveis & Frequência & $\%$ \\
\hline Malformação fetal & 19 & 17,1 \\
Doença hiperfensiva específica da gestação & 12 & 10,8 \\
Descolamento prematuro de placenta & 11 & 9,9 \\
Achados sugestivos de infecção materna & 8 & 7,2 \\
Alterações do cordão & 6 & 5,4 \\
Diabefes melliłus gestacional & 5 & 4,5 \\
Outros achados & 12 & 10,8 \\
Sem achados & 38 & 34,3 \\
Total & 111 & 100,0 \\
\hline
\end{tabular}

Tabela 4. Comparação entre os diagnósticos do estudo dos fetos e anexos com a avaliação clínica

\begin{tabular}{|c|c|c|c|}
\hline \multirow[b]{2}{*}{ Avaliação clínica } & \multicolumn{3}{|c|}{$\begin{array}{c}\text { Estudo dos fetos e anexos } \\
\text { n (\%) }\end{array}$} \\
\hline & $\begin{array}{l}\text { Causa do } \\
\text { óbito fetal } \\
\text { identificada }\end{array}$ & $\begin{array}{l}\text { Causa do } \\
\text { óbito fetal não } \\
\text { identificada }\end{array}$ & Total \\
\hline Causa do óbito fetal identificada & $54(48,6)$ & $19(17,2)$ & $73(65,8)^{\star}$ \\
\hline Causa do óbito fetal não identificada & $20(18,0)$ & $18(16,2)$ & $38(34,2)$ \\
\hline Total & $74(66,6)^{*}$ & $37(33,4)$ & $111(100,0)$ \\
\hline
\end{tabular}

${ }^{*} p=0,88 ; \mid C 95 \% 0,5-1,8$ insuficiência placentária $(32,4 \%, \mathrm{n}=23)$, RCIU $(16,9 \%$, $\mathrm{n}=12)$ e corioamnionite $(12,7 \%, \mathrm{n}=9)$.

\section{Discussão}

A população estudada apresentou características semelhantes à de outros estudos ${ }^{6,16,17}$ sobre mortalidade fetal no País - média de 23,9 $( \pm 6,4)$ anos, maioria branca e casada ou vivendo em união estável. Quase metade das pessoas estudadas tinha o ensino fundamental incompleto e a maioria, uma ou nenhuma gestação prévia.

Em um estudo longitudinal demonstrou-se redução importante nas perdas fetais tardias (definida como $>28$ semanas de gestação) nas últimas décadas, resultado de estratégias que foram desenvolvidas para intervir em questões específicas obstétricas ${ }^{18}$. Os dados encontrados infelizmente são discordantes, pois aproximadamente dois terços $(63,0 \%)$ das mortes aconteceram em gestações tardias, realidade intrigante se for levado em consideração que a cobertura pré-natal atende quase $100 \%$ das usuárias do sistema de saúde vigente no município, com média de consultas de $6,2 \%{ }^{19}$.

A Portaria $\mathrm{n}^{\circ} 72$ do MS, de 11 de janeiro de 2010, estabelece que a vigilância do óbito infantil e fetal é obrigatória nos serviços de saúde (públicos e privados) que integram o Sistema Único de Saúde (SUS). Deve ser realizada com os dados retirados da DO, documento oficial que atesta a morte de um indivíduo, com todos os campos adequadamente preenchidos para que o Sistema de Informação sobre Mortalidade (SIM), do MS, alimente sua base de dados e permita a investigação dos óbitos ${ }^{7,9}$.

Diante do exposto, era de se esperar que facilmente se obtivessem dados concretos para estudar o desfecho óbito fetal analisando os dados da DO, mas na prática não se observa tal situação, visto que neste estudo em aproximadamente metade das DOs há a assertiva "na dependência de exames complementares" na linha em que deveria constar a causa determinante da morte. $\mathrm{Na}$ literatura, um estudo relatou que $53,3 \%$ das mortes fetais não tinham causas determinadas em $\mathrm{DO}^{6}$. Pesquisas envolvendo os dados extraídos das declarações reforçam as dificuldades decorrentes da falta de informações ${ }^{9,20}$. Vale lembrar que a DO é documento de preenchimento obrigatório pelos médicos, com atribuições e responsabilidades detalhadas pela Resolução $n^{\circ} 1.779$ de 2005 do Conselho Federal de Medicina ${ }^{7}$.

Sabe-se que as causas associadas ao óbito fetal são muitas, o que dificulta melhor definição da sua etiologia, bem como é necessário estudar a importância relativa dos diferentes motivos em diferentes populações ${ }^{16}$. Na população estudada, os achados da autópsia do feto e anatomopatológico dos anexos, usados no estudo como padrão de referência, chamam a atenção, pois se referem, em grande parte, a aspectos que poderiam ser 
detectados no acompanhamento da gestação. Mais de $30 \%$ das possíveis causas estão relacionadas à adequada assistência pré-natal — infecção e alterações placentárias. Embora o objetivo da realização de autópsias seja aprimorar a informação sobre as causas de morte, $33 \%$ dos óbitos ficaram sem um motivo definido, mostrando que sua realização não tem melhorado a qualidade das informações? .

$\mathrm{Na}$ avaliação clínica, 65,8\% dos achados apresentaram-se como potencial causa. Malformações $(26,0 \%)$, infecção materna $(11,0 \%)$ e DPP $(15,1 \%)$ foram encontradas na avaliação clínica e no estudo do feto e anexos. Já a DHEG $(16,4 \%)$ e o $\mathrm{DMG}(6,9 \%)$ foram possíveis motivos de óbitos relacionados a complicações da gestação registradas somente nos dados clínicos. Comparadas as percentagens entre os dois grupos, verificou-se que complicações da placenta (DPP) e do cordão umbilical foram mais frequentes nos achados clínicos, possivelmente devido à ausência de repasse das informações da gestação e do parto ao patologista no momento da realização da necropsia. A análise torna-se mais difícil quando a necropsia é feita pelo Serviço de Verificação de Óbito (SVO), pois a placenta não acompanha o feto e as avaliações acontecem separadamente?

Em uma revisão sistemática, Corabian et al. ${ }^{13}$ demonstraram que achados da necropsia fetal concordavam com o diagnóstico clínico em 28,6 a 89,8\% dos casos e em 10,2 a 38,0\% revelaram novo diagnóstico ou levaram à alteração da hipótese clínica, ou seja, a necropsia foi diagnóstica. Informações adicionais, que não alteraram o diagnóstico clínico, foram providas pela necropsia em 3,9 a $24,3 \%$ dos casos. No entanto, a causa de óbito permanecia não explicada em até $40 \%$ dos casos. Quando acrescida da análise anatomopatológica da placenta, havia concordância com achados clínicos e/ou da necropsia em até $75 \%$ dos casos e eram diagnósticas em 22,7 a 46,3\% do total. O motivo do óbito permanecia não explicado em apenas $12 \%{ }^{13}$.

Já neste estudo, a proporção de diagnósticos entre o exame anatomopatológico e a avaliação clínica não apresentou diferença significativa. Ambas encontraram potencial causa em $48,7 \%$ das análises. Entretanto, a adição do exame anatomopatológico identificou 20 motivos não detectados pela avaliação $(18,0 \%)$, a qual, em contrapartida, verificou $19(17,1 \%)$ isoladamente. Desta maneira, a associação de ambas as modalidades permitiu a redução para $16,2 \%$ dos casos indefinidos.

Revisões sistemáticas destacam o valor da investigação dos possíveis fatores envolvidos no óbito, como história obstétrica, história familiar e pré-natal atual $^{13,14}$. A autópsia e o exame da placenta fornecem informações valiosas, sendo considerados essenciais na determinação da causa e recomendados por todas as diretrizes $^{1,11,13,14}$. Infelizmente, pode haver dificuldades com a autorização dos pais para o estudo do feto ${ }^{21}$. Torna-se, assim, importante trabalhar com os pais na aceitação desse procedimento. Diversas abordagens facilitam esta aceitação, entre elas destaca-se a sensibilidade durante a discussão da avaliação fetal e a informação de que a aceitação pode ter impacto positivo em uma gestação futura $^{22}$. Mesmo quando negada, deve-se oferecer o estudo anatomopatológico da placenta, que parece ser uma opção importante na investigação ${ }^{13}$.

Gardosi et al. ${ }^{11}$, ao comparar duas classificações, encontrou que $85 \%$ dos casos foram atribuídos a condições relevantes quando utilizado o sistema ReCoDe, permanecendo apenas $15 \%$ como "não classificado". Em contrapartida, quando usou-se o de Wigglesworth, $66 \%$ dos casos mantiveram-se não explicados, podendo dar a falsa impressão de que se tratam de casos não evitáveis. O autor observou que a condição mais comum foi a restrição de crescimento fetal $(43 \%)^{11}$. Similarmente, no presente estudo, quando usada a classificação ReCoDe, apenas 9,9\% dos casos foram atribuídos à categoria "não classificado" e 32,5\% à de restrição de crescimento fetal.

Ao comparar-se os resultados do ReCoDe com a determinação da causa do óbito determinada pela clíni$\mathrm{ca} /$ patologia, verifica-se que essa ferramenta contribuiu para o esclarecimento da causa mortis em 38,9\% dos casos nos quais ambas as modalidades não foram capazes de apontar o motivo, muito embora houvessem identificado e registrado.

Ressalta-se, com o levantamento, a importância do sistema $\mathrm{ReCoDe}$, o qual permitiu classificar mais de $90 \%$ desta casuística, uma vez que qualquer sistema classificatório tem por objetivo primordial ajudar o clínico na identificação das causas e fatores associados.

O estudo apresentou limitações decorrentes pelo fato de a análise dos dados ser retrospectiva, resultando na ausência e/ou não uniformização deles nos prontuários, e pela falta, nas instituições, de um protocolo de investigação. Visando solver tais aspectos em futuros estudos, propõe-se a criação de um padrão de investigação de OF usando-se parâmetros para os registros dos dados clínicos e os achados anátomo-patológicos. Deve ser imprescindível que as instituições públicas e privadas que prestam assistência às mulheres mantenham fluxogramas que comtemplem estudo do feto e dos anexos.

Recomendamos a criação de um protocolo de investigação do óbito ou adequação dos inúmeros já existentes, e o uso de um fluxograma bem estabelicido, que ordene os passos da investigação e seja conhecido pela equipe que presta assistência.

Os médicos devem ser capacitados para o adequado preenchimento da DO, reforçando o valor legal e ético 
dessa atividade. $\mathrm{E}$ os pais precisam receber explicações sobre o valor e o beneficio potencial da investigação do óbito, obtendo o consentimento para a autópsia do feto. Isso é responsabilidade dos médicos, enfermeiros e equipe envolvida com a assistência ${ }^{13}$.
Finalmente, é preciso uniformizar a investigação nos serviços governamentais para garantir que os detalhes não sejam perdidos, criando uma busca ordenada e sistemática para a causa da morte ${ }^{11,13}$ e um sistema classificatório mais abrangente, como o ReCoDe.

\section{Referências}

1. Bonetti LR, Ferrari P, Trani N, Maccio L, Laura S, Giuliana S, et al. The role of fetal autopsy and placental examination in the causes of fetal death: a retrospective study of 132 cases of stillbirths. Arch Gynecol Obstet. $2011 ; 283(2): 231-41$

2. Maternal-Fetal Medicine Committee, Clinical Practice Obstetrics Committee, Leduc L, Farine D, Armson BA, Brunner M, Crane J, Delisle MF, et al. Stillbirth and bereavement: guidelines for stillbirth investigation. J Obstet Gynaecol Can. 2006;28(6):540-52.

3. Cousens S, Blencowe H, Stanton C, Chou D, Ahmed S, Steinhardt $L$, et al. National, regional, and worldwide estimates of stillbirth rates in 2009 with trends since 1995: a systematic analysis. Lancet. $2011 ; 377(9774): 1319-30$.

4. Brasil. Ministério da Saúde. DATASUS [Internet]. Informações de Saúde. Notas técnicas: óbitos fetais - Brasil, 2009. Brasília (DF): Ministério da Saúde; 2009. [citado 2011 Out 30]. Disponível em: <http://tabnet.datasus.gov.br/cgi/deftohtm. exe? sim/cnv/fetlouf.def>

5. Frøen JF, Cacciatore J, McClure EM, Kuti O, Jokhio AH, Islam $M$, et al. Stillbirths: why they matter. Lancet. $2011 ; 377(9774)$ : 1353-66.

6. Andrade LG, Amorim MM, Cunha AS, Leite SR, Vital SA. [Factors associated with stillbirth in a school maternity in Pernambuco: a case control study]. Rev Bras Ginecol Obstet. 2009;31(6):285-92. Portuguese.

7. Brasil. Ministério da Saúde. Secretaria de Atenção à Saúde. Manual de vigilância do óbito infantil e fetal e do Comitê de prevenção do óbito infantil e fetal. 2a ed. Brasília (DF): Ministério da Saúde; 2009.

8. Lawn JE, Gravett MG, Nunes TM, Rubens CE, Stanton C; GAPPS Review Group. Global report on preterm birth and stillbirth (1 of 7): definitions, description of the burden and opportunities to improve data. BMC Pregnancy Childbirth. 2010;10 Suppl 1:S1.

9. Almeida MF, Alencar GP, Schoeps D, Minuci EG, Silva ZP, Ortiz LP, et al. Qualidade das informações registradas nas declarações de óbito fetal em São Paulo, SP. Rev Saúde Pública. 201 1;45(5):845-53.

10. Smith GC, Fretts RC. Stillbirth. Lancet. 2007;370(9600):1715-25.
11. Gardosi J, Kady SM, McGeown P, Francis A, Tonks A. Classification of stillbirth by relevant condition at death (ReCoDe): population based cohort study. BM. 2005;331 (7525): $11113-7$.

12. Silver RM, Heuser CC. Stillbirth workup and delivery management. Clin Obstet Gynecol. 2010;53(3):681-90.

13. Corabian P, Scott NA, Lane C, Guyon G. Guidelines for investigating stillbirths: an update of a systematic review. J Obstet Gynaecol Can. 2007;29(7):560-7.

14. Silver RM, Heuser CC. Stillbirth workup and delivery management. Clin Obstet Gynecol. 2010;53(3):681-90.

15. Organização Mundial da Saúde. CID-10: classificação estatística internacional de doenças e problemas relacionados à saúde. $8^{a}$ ed. São Paulo: Edusp; 2000.

16. Aquino MMA, Cecatti JG. [Epidemiology of fetal death in a low income population]. Rev Bras Ginecol Obstet. 1998;20(2):71-5. Portuguese.

17. Vardanega K, De Lorenzi DRS, Spiandorello WP, Zapparoli MF. [Risk factors for stillbirth at a universitary hospital in Southern Brazil]. Rev Bras Ginecol Obstet. 2002;24(9):617-22. Portuguese.

18. Fretts R. Stillbirth epidemiology, risk factors, and opportunities for stillbirth prevention. Clin Obstet Gynecol. 2010;53(3):588-96.

19. Vieira MS, Patrício ZM, Santos ML. A assistência pré-natal prestada à gestante em serviços de saúde de Florianópolis-SC, buscando a qualidade com foco na normatização preconizada e nas necessidades das mulheres [dissertação]. Florianópolis: Universidade Federal de Santa Catarina; 2005.

20. Almeida MF, Alencar GP, Novaes HMD, Ortiz LP. Sistemas de informação e mortalidade perinatal: conceitos e condições de uso em estudos epidemiológicos. Rev Bras Epidemiol. 2006;9(1):56-68.

21. Lawn J, Shibuya K, Stein C. No cry at birth: global estimates of intrapartum stillbirths and intrapartum-related neonatal deaths. Bull World Health Organ. 2005;83(6):409-17.

22. ACOG Practice Bulletin No. 102: management of stillbirth. Obstet Gynecol. 2009;1 13(3):748-61. 